\section{SEVERITY OF ILLNESS}

\section{Critically III Patients}

Patients requiring ventilatory and/or circulatory support, non-invasive ventilation, invasive mechanical ventilation, or ECMO

Moderately III Patients Patients newly requiring low-flow supplemental oxygen

\title{
RECOMMENDATIONS
}

Dexamethasone $6 \mathrm{mg} \mathrm{PO/IV} \mathrm{daily} \mathrm{for} 10$ days (or until discharge if sooner) is recommended.

Tocilizumab is recommended for patients who are on recommended doses of dexamethasone therapy (or a dose-equivalent corticosteroid) AND are within 14 days of nosocomially acquired).

RECOMMENDATIONS FOR DRUG SHORTAGE SITUATIONS

- In drug shortage situations, a single dose of tocilizumab $400 \mathrm{mg}$ IV or sarilumab $400 \mathrm{mg}$ iV should be used for all elieible patients. A second dose of tocilizumab or sarilumab should not be given to any patient.

Baricitinib $4 \mathrm{mg} \mathrm{PO} / \mathrm{NG}$ daily for 14 days (or until discharge if sooner) is therapy (or a dose-equivalent corticosteroid) or who have a contraindication to corticosteroid treatment. The panel does not recommend combined use of

Dexamethasone $12 \mathrm{mg}$ PO/IV daily for 10 days (or until discharge mer) may sarilumab) or baricitinit. This recomable to receive IL-6 in pitor. cilizumab, sarilumab) or baricitinib. This recommendation is bact inpatient treatment options with a reason with a reason
to the vicror variant

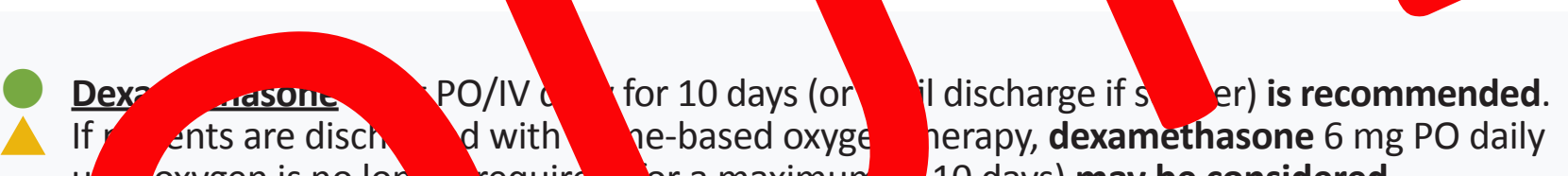

d with he-based oxyge 4 oxygen is no lon, equire pr a maximur 10 days) may be considered. E desivir $200 \mathrm{mg}$ IV ay 1 , the aily for 4 days is recommended.

$\Delta$ Th eutic dose antic ulation may be considered over prophylactic dose All oth lation in pat sh who are felt to be at low risk of bleeding. - SARS-CoV-2 neutralizing antibodies are not recommended for moderately ill patients. sotrovimab on page 2 .

Go to page 2 for recommendations in mildly ill patients
Prophylactic dose low molecular weight or unfractionated heparin is recommended. These patients should not receive therapeutic dose anticoagulation in unless they have separate indication for this treatment.

- Remdesivir is not recommended for patients receiving $m e$ nical ventilatio

Remdesivir $200 \mathrm{mg}$ IV on day 1 , then 100 mo $y$ for 4 o may be consid. cannula, or non-invasive mech by high-flow $n$

SARS-CoV-2 neutralin nodies are recom ded for for sotrovimab

$\square$
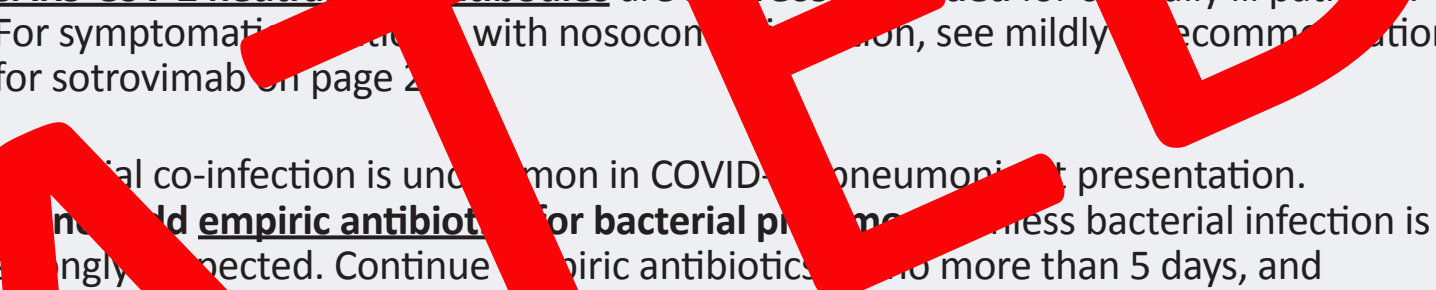

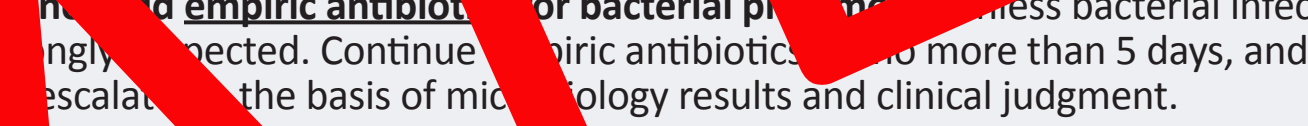

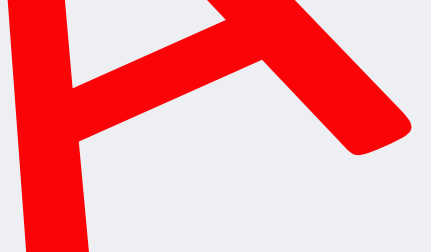

Tocilizumab is recommended for patients who have evidence of systemic disease progression (i.e., increasing oxygen or ventilatory requirements) despite 24-48 hours of recommended doses of dexamethasone therapy (or a dose-equivalent
corticosteroid), AND are within 14 days of hospital admission (or within 14 days of a corticosteroid), AND are within 14 days of hospital admission (or with
new COVID-19 diagnosis if the infection was nosocomially acquired).

RECOMMENDATIONS FOR DRUG SHORTAGE SITUATIONS
In drug shortage situations, a single dose of tocilizumab $400 \mathrm{mg}$ IV or
sarilumab $400 \mathrm{mg}$ IV should be used for all eligible patients. A second dose of
tocilizumab or sarilumab should not be given to any patient.
Baricitinib $4 \mathrm{mg}$ PO/NG daily for 14 days (or until discharge if sooner) is
recommended in patients who are on recommended doses of dexamethasone
therapy (or a dose-equivalent corticosteroid) or who have a contraindication
to corticosteroid treatment. The panel does not recommend combined use of
baricitinib and IL-6 inhibitors due to absence of safety and efficacy evidence.

CURRENTLY NOT

RECOMMENDED*

There is insufficient evidence to support the use of the
following therapies in the treatment of COVID-19 outsid of clinical trials or where othe ndications would justify its use

Colchicine

Interferon (with or withou ribavirin)

$\checkmark$ Vitamin D

RECOMMENDED AGAINST*

The following therapies are no COVID-19 due to lack of benefit potential harm, and system

Antibiotics (azithromycin)

Casirivimab-imdevimab

Omicron variant

Hydroxychloroquine or

oroquine

Ivermectin

Lopinavir/ritonavir

*Applies to patients with any

serity of illness 


\section{Mildly III Patients}

Patients who do not require new or their baseline

\section{Tier 1}

Immunocompromised individuals ${ }^{1}$ not expected to mount an adequate immune response to COVID-19 vaccination or SARS-CoV-2 infection due to their underlying conditions,
regardless of vaccine status: $O R$ Unvaccinated ${ }^{2}$ individuals at highest risk of severe disease (only if also age $\geq 70$ years, Indigenous and age $\geq 60$ years, or age $\geq 60$ years with
one or more risk factors

immunocompromised individuals are at higher risk, and should be prioritized for treatment in this tier.'

\section{Tier 2}

Unvaccinated ${ }^{2}$ individuals at risk of severe disease (only flso age $\geq 60$ or $\geq 50$ years with one or more risk factors $)^{3} .^{4}$

\section{Tier 3}

Vaccinated individuals at highest risk of severe disease (only if also age $\geq 70$ years, Indigenous and age $\geq 60$ years, factors ${ }^{3}$ ). Vaccinated individuals who are $>6$ months from their last dose of vaccine are at higher risk, and should

\section{Tier 4}

Vaccinated individuals at risk of severe disease (only if also age $\geq 60$ years, Vars with one or mo risk factors ${ }^{3}$. Vaccinated individuals who are $>6$ monthis their last dose of vaccin prioritized for treatment in this tier.
This guidance applies to mildly ill patients in any setting, including the community, hospital (including nosocomial cases), and congregate care settings. It is recommended that eligibility for outpatient therapies include patients who test positive for SARS-CoV-2 on either PCR or a healthcare-professional administered RAT or ID Now.

\section{RECOMMENDATIONS}

Sotrovimab $500 \mathrm{mg}$ IV $\times 1$ dose is recommended for these patients if they present within 7 days of symptom onset.

- The individuals should have a reasonable expectation for 1-year survival prior to SARS-CoV-2 infection. It is recommended that monoclonal antibody therapy be administered to non-hospitalized
integrated healthcare services, community paramedicine, and outpatient infusion clinics.

HIGHER RISK OF
SEVERE DISEASE

Tier 1

Tier 2

If sotrovimab is unavailable or contraindicated:

Remdesivir $200 \mathrm{mg}$ IV on day 1 , then $100 \mathrm{mg}$ IV daily for 2 days may be considered for these patients if they - 11 within 7 dac symptom onset and: (1) more effective - These individuals should have a reasonable expectation for 1-year survival prior to SARS-Coly barmier.
ction.

$\longrightarrow$ If remdesivir is unavailable or contraindicated:

Fluvoxamine may be considered for patients with mild COVID-19 illn ontin thin 7 days of sympt onset. The re imended starting dose is $50 \mathrm{mg}$ PO daily, the onsultation a putn 7 provider interactions with fluvoxamine. This recommendation bala
options for mild illness with a reasonable safety profile dumg a surg $\begin{gathered}\text { low certainty } \\ \text { COVID-19 cas ue to the Omicron }\end{gathered}$

tow certainty evidence of reduction in duration of symptoms, and the need for ou treatment options a reasonable ty profi' ing an anticipated spike in COVID-19 cases due to the Omicron

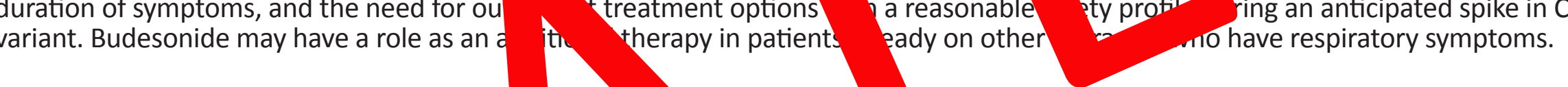

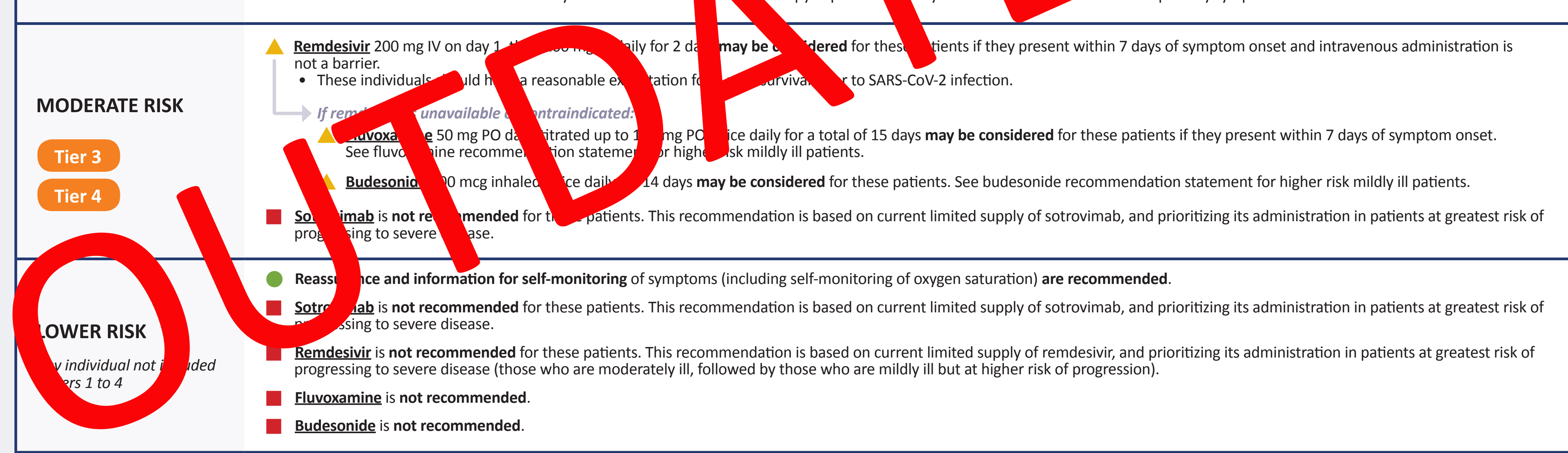

There is currently insufficient evidence to make a recommendation around aspirin or anticoagulation for mildly ill patients.

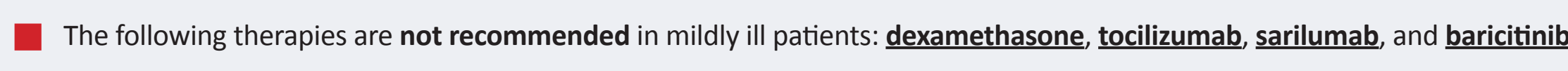

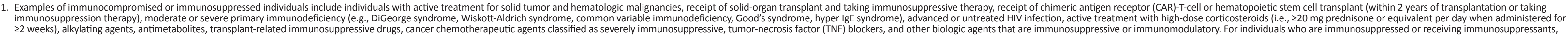

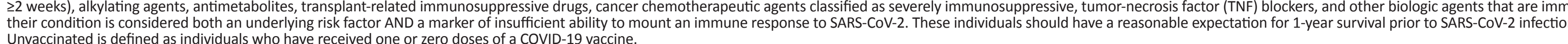

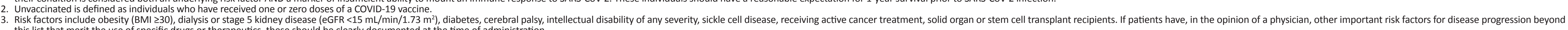

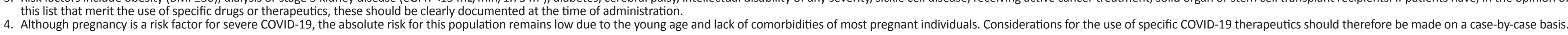

